\title{
Keck spectroscopic survey of strongly lensed galaxies in Abell 1703: further evidence of a relaxed, unimodal cluster
}

\author{
J. Richard ${ }^{1,2, \star}$, L. Pei ${ }^{2}$, M. Limousin ${ }^{3,4}$, E. Jullo ${ }^{5}$, and J. P. Kneib \\ 1 Durham University, Physics and Astronomy Department, South Road, Durham DH1 3LE, UK \\ e-mail: johan.richard@durham.ac.uk \\ 2 Caltech Astronomy, MC105-24, Pasadena, CA 91125, USA \\ 3 Laboratoire d'Astrophysique de Toulouse-Tarbes, Université de Toulouse, CNRS, 57 avenue d'Azereix, 65000 Tarbes, France \\ ${ }^{4}$ Dark Cosmology Centre, Niels Bohr Institute, University of Copenhagen, Juliane Marie Vej 30, 2100 Copenhagen, Denmark \\ 5 Laboratoire d'Astrophysique de Marseille, OAMP, CNRS-Université Aix-Marseille, 38 rue Frédéric Joliot-Curie, \\ 13388 Marseille Cedex 13, France
}

Received 17 November 2008 / Accepted 30 January 2009

\begin{abstract}
Context. Strong gravitational lensing is a unique tool that can be used to accurately model the inner mass distribution of massive galaxy clusters. In particular, clusters with large Einstein radii provide a wealth of multiply imaged systems in the cluster core. Measuring the redshift of these multiple images provides strong constraints for precisely determining the shape of the central dark matter profile.

Aims. This paper presents a spectroscopic survey of strongly lensed galaxies in the massive cluster lens Abell 1703, which displays a large Einstein radius $\left(28^{\prime \prime}\right.$ at $\left.z=2.8\right)$ and numerous strongly-lensed systems including a central ring-like configuration.

Methods. We used the LRIS spectrograph on Keck to target multiple images and lensed galaxy candidates, and used the measured spectroscopic redshifts to constrain the mass distribution of the cluster using a parametric model.

Results. The spectroscopic data enable us to measure accurate redshifts for 7 sources at $z>2$, all of which are in good agreement with their photometric redshifts. We update the identification of multiply imaged systems by discovering 3 new systems and identifying a radial counter image. We also report the discovery of a remarkably bright $\sim 3.6 L^{*} i$-band dropout at $z=5.827$ in our mask and it is only moderately magnified by the cluster $(\mu \sim 3.0 \pm 0.08)$. The improved parametric mass model, including 16 multiple systems with 10 spectroscopic redshifts, further constrains the smooth cluster-scale mass distribution with a generalized NFW profile of best-fit logarithmic slope $\alpha=0.92 \pm 0.04$, concentration $c_{200}=4.72 \pm 0.40$ and scale radius $r_{\mathrm{s}}=476 \pm 45 \mathrm{kpc}$. The overall rms in the image plane is $1.3^{\prime \prime}$.

Conclusions. Our strong-lensing model allows us to predict a large-scale shear signal that is consistent with weak-lensing measurements inferred from Subaru data out to $4 \mathrm{Mpc} h^{-1}$. Also considering the strong-lensing modeling requires a single dark matter clump, this suggests that Abell 1703 is a relaxed, unimodal cluster. This unique cluster could be probed further using deep X-ray, SZ, and dynamics analysis for a detailed study of the physics in a relaxed cluster.
\end{abstract}

Key words. galaxies : high-redshift - galaxies: distances and redshifts - galaxies: clusters: individual: Abell 1703 gravitational lensing

\section{Introduction}

Massive galaxy clusters with large Einstein radii $\left(R_{\mathrm{E}} \gtrsim 20^{\prime \prime}\right)$ offer a wealth of information about their central mass distribution by displaying large numbers of multiple images in their stronglensing regions. The most striking case is the cluster Abell 1689, with $R_{\mathrm{E}} \sim 50^{\prime \prime}$ (Broadhurst et al. 2005; Limousin et al. 2007), which holds the largest number of strong lensing constraints in a single field. This gives an opportunity to map inner-mass profiles to a high level of precision. Parametric cluster-lens mass models have been widely used (Kneib et al. 1996; Smith et al. 2005; Richard et al. 2007), but accurate redshift information is necessary to construct accurate mass models and obtain their fiducial parameters. Only one redshift of a set of multiple images enables us to estimate the absolute total mass within the Einstein radius. Adding many more redshifts allows us to measure the full central mass profile accurately. Even though photometric

^ Marie-Curie fellow. redshifts offer an acceptable level of precision for such modeling (Broadhurst et al. 2005; Bradač et al. 2008), spectroscopic redshifts are the most reliable way to confirm the identifications and find new multiply-imaged systems. Lowering the redshift error on the multiple systems with spectroscopy has another tool, because one can use strong-lensing to constrain the cosmological parameters $w$ and $\Omega_{\mathrm{m}}$ based on purely geometrical considerations (Link \& Pierce 1998; Golse et al. 2002).

On the theoretical side, Broadhurst \& Barkana (2008) have compared the measured lensing parameters for a sample of clusters with large $R_{\mathrm{E}}$ to predictions from the standard $\Lambda \mathrm{CDM}$ cosmology, and report a 4- $\sigma$ discrepancy in the existence of such large $R_{\mathrm{E}}$ for the corresponding virial mass. Their result, based on a sample of massive halos derived from the Millennium simulation, includes a full treatment of the projection effects, which strongly bias this sample towards alignments of the triaxial darkmatter halos along the line of sight. This bias is also pointed out by Oguri \& Blandford (2009), who investigated a full-sky 
Monte-Carlo realizations of massive clusters. They show that, although the population of clusters with large Einstein radii is scarce, it is possible to find Einstein radii as large as $50^{\prime \prime}$ in the whole sky, under the assumptions of a WMAP5 cosmology. The main difference beween Broadhurst \& Barkana (2008) and Oguri \& Blandford (2009) is that the latter treats the sample as a whole-sky survey, which is far more representative of the observed population than the Millennium simulation, which only covers a small volume of $\sim 5000 \mathrm{Mpc}^{3}$. This undermines any disagreement between the presence of large $R_{\mathrm{E}}$ and $\Lambda$-CDM.

Among the clusters with large $R_{\mathrm{E}}$, we have started to study the mass distribution of Abell 1703, in Limousin et al. (2008), hereafter L08. This is one of the richest clusters discovered in the Sloan Digital Sky Survey (SDSS), and it shows strong gravitational lensing (Hennawi et al. 2008). In this previous work, we identified 13 systems forming highly-magnified multiple images, including a central ring. Based on the spectroscopic redshift of this central feature and photometric redshifts for the remaining images, all the lensing systems have been reproduced by a single NFW (Navarro et al. 1997) profile for the dark matter, making Abell 1703 relatively simpler than other bimodal lensing clusters (e.g. Elíasdóttir et al. 2007 for Abell 2218; Richard et al. 2007 for Abell 68; Verdugo et al. 2007 for MS2053). We therefore decided to use it as a more reliable probe for comparing the results of strong and weak-lensing measurements in clusters with large Einstein radii from Broadhurst et al. (2008).

We present in this paper results from a spectroscopic survey conducted at Keck using the LRIS instrument, aiming at confirming the photometric redshifts, multiple images identification, and precisely constraining the mass distribution in Abell 1703. We obtained new spectroscopic redshift measurements for most of the systems identified by L08 (10 out of 13), in good agreement with their corresponding photometric redshifts. We also report an exceptionally bright $i$-band dropout at $z \sim 6$, forming a magnified single image close to the region of multiple images. We further identify three new systems of multiple images and constrain the lensing model, as well as comparing the weak-lensing extrapolation of our model to the recently measured weak-lensing profile of Broadhurst et al. (2008).

We present the observations and data reduction of the spectroscopic data in Sect. 2. Section 3 gives the redshift measurements, spectra, and the main results from the mass modeling. We discuss these results in Sect. 4. Throughout the paper, we used a standard $\Lambda$-CDM model with $\Omega_{\mathrm{m}}=0.3, \Omega_{\Lambda}=0.7$, and $H_{0}=70 \mathrm{~km} \mathrm{~s}^{-1} \mathrm{Mpc}^{-1}$, whenever necessary. In this cosmology, $1^{\prime \prime}$ on the sky is equivalent to a physical distance of $4.244 \mathrm{kpc}$ at the redshift $z=0.28$ of the cluster. All magnitudes are quoted in the AB system.

\section{Observations}

\subsection{Imaging and photometric redshifts}

The current study is based on the same imaging data as described in L08, based on Hubble Space Telescope and Subaru observations of Abell 1703 covering broad-band filters from $B$ to $H$. These images were previously used to derive photometric redshifts with the software Hyperz (Bolzonella et al. 2000). We briefly summarize the characteristic parameters, central wavelength, and depth, of these images in Table 1.
Table 1. Summary of the broad-band images used for the photometry.

\begin{tabular}{llcc}
\hline \hline Filter & $\lambda(\mu \mathrm{m})$ & Exposure time $(\mathrm{ksec})$ & Depth $^{(*)}(\mathrm{AB})$ \\
\hline$A C S / F 435 W$ & 0.435 & 7.05 & 26.5 \\
$A C S / F 475 W$ & 0.480 & 5.56 & 27.5 \\
$A C S / F 555 W$ & 0.539 & 5.56 & 27.1 \\
$A C S / F 625 W$ & 0.635 & 8.49 & 27.6 \\
$A C S / F 775 W$ & 0.779 & 11.13 & 27.3 \\
$A C S / F 850 L P$ & 0.908 & 17.8 & 26.9 \\
NICMOS $/ F 110 W$ & 1.147 & 0.26 & 25.4 \\
MOIRCS $/ H$ & 1.642 & 18.87 & 25.3 \\
\hline
\end{tabular}

(*) Given as the $5 \sigma$ detection limit for a point source.

\subsection{Keck spectroscopy}

We used the Low Resolution Imager and Spectrograph (LRIS, Oke et al. 1995) on the Keck telescope to perform multi-slit observations of the Abell 1703 cluster field. We designed a multislit mask containing 32 slits of $1.0^{\prime \prime}$ width to include as many of the multiple systems previously identified in L08 as possible, with a few tilted slits following the geometry of long arcs (Fig. 1). In the case of fainter or less reliable identifications, independent images of the same system were observed in separate slits. Additional slits in the mask included new multiple systems candidates, cluster members, and background galaxies as estimated through their photometric redshifts.

The LRIS instrument was set up with a $6800 \AA$ dichroic isolating the blue arm, with a 300-line/mm grism blazed at $5000 \AA$, from the red arm, with a 600 -line/mm grating blazed at $7500 \AA$. This ensures a high throughput over the wavelength range 3500 to $9500 \AA$ and good spectral resolution in the red to resolve skylines as well as [OII] emission line doublets in the redshift range 0.85-1.40. This instrument setup is also well-matched with the photometric redshift estimates of the targeted sources. We obtained $2 \mathrm{~h}$ of exposure time on 2008 May 10 in photometric conditions and very good seeing $\left(0.7^{\prime \prime}\right)$.

\subsection{Data reduction and redshift measurements}

Data reduction of the spectra was performed using the Python version of the Kelson (2003) reduction scripts, which offer the advantage of processing the images in their distorted framework. This helps to reduce noise correlations, in particular for the case of tilted slits. We performed standard reduction steps for bias removal, flat-field correction, wavelength calibration, sky subtraction, and cosmic-ray rejection, and used observations of the standard star BD+33-2642 obtained on the same night to derive the flux calibration.

Close-ups on the targets and extracted spectra are presented in Figs. 2 and 3. We measured the redshift for 33 sources in the entire LRIS mask. The spectra show either Lyman- $\alpha$ (in absorption or emission) with additional ultraviolet absorption lines in the blue part or a resolved doublet of [OII]) in the red part of the spectrum. In two multiple systems (10/11 and 16), we observed these images in multiple slits: redshift measurements were obtained by identifying significant absorption lines after stacking the relevant exposures. For each spectrum, the average redshift value is obtained from the peaks of the main spectral features identified, while the corresponding error is taken from the spectral dispersion. Additional uncertainties generated by the 


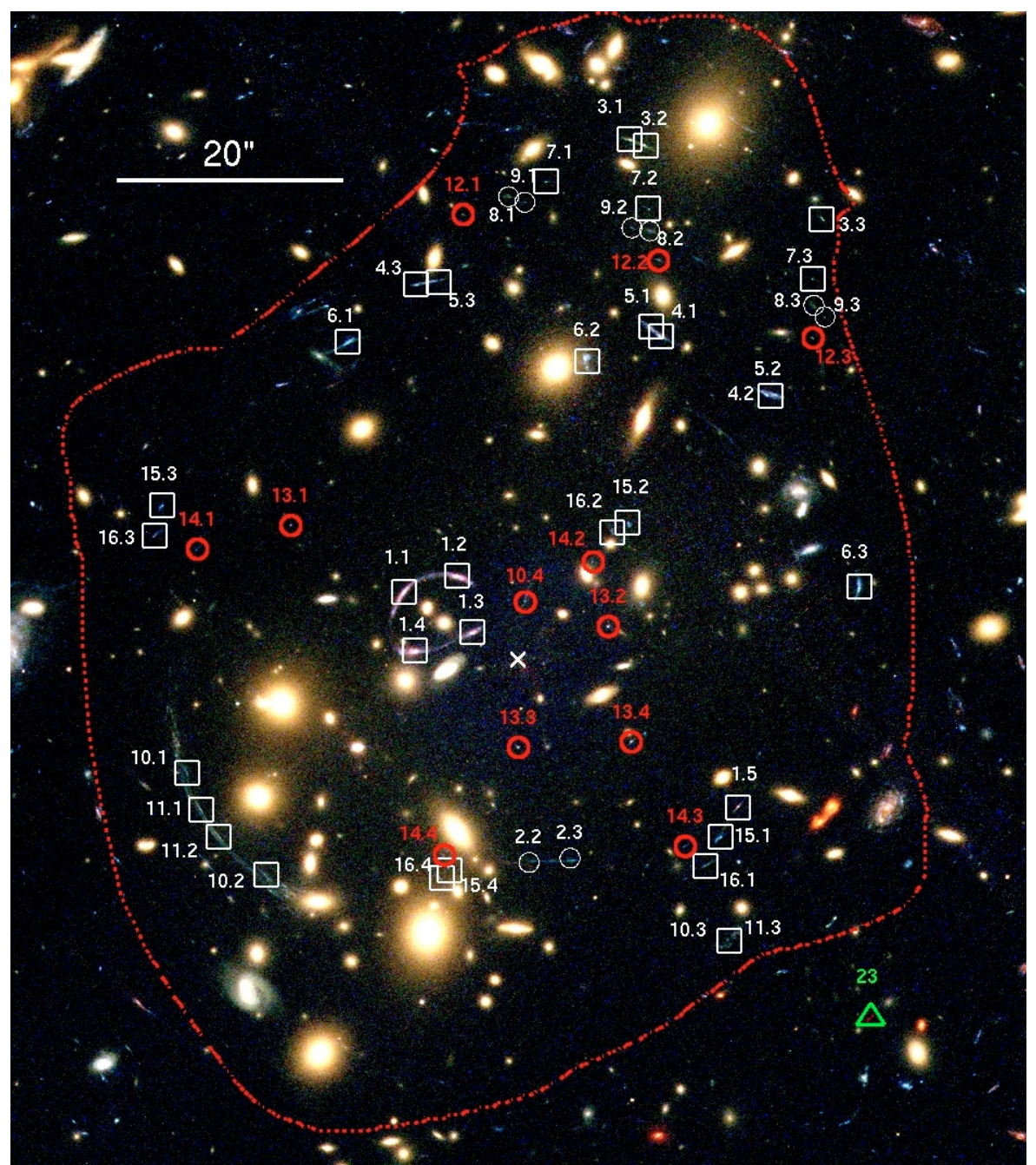

Fig. 1. ACS color image of Abell 1703 (combination of the $H S T$ filters $F 450 W, F 606 W$ and $F 850 L P$ ), showing the location of all the multiplyimaged systems included in this study. The white cross at the center of the image marks the location of the brightest cluster galaxy, which has been subtracted for clarity. The dashed line outlines the limit of the region where we expect multiple images up to $z=6$. The systems presented in L08 are shown in white, and the new systems presented in this work are shown in red. Squares identify the multiple images with spectroscopic redshifts.The green triangle located to the lower right marks the location of the $i$-band dropout presented in Sect. 3.2.

accuracy of the relative and absolute wavelength calibrations, about 1.1 and $1.5 \AA$, respectively, were quadratically added to yield the final redshift errors. A confidence class $z_{\text {class }}$, ranging from 1 to 4 , was assigned to each measurement according to the prescription of Le Fevre et al. (1995), which corresponds to a probability level for a correct identification of 50\%, 75\%, 95\%, and $100 \%$, respectively. A specific value of 9 is used when only a single secure spectral feature is seen in emission.

Table 2 summarizes the redshift measurements $z_{\text {spec }}$, confidence class $z_{\text {class }}$ and the spectral features used to derive the redshift for all objects detected in the LRIS mask, including those additional targets serendipitously falling into the slits. For the majority of sources located within the ACS field of view, we report the corresponding photometric redshift $z_{\text {phot }}$ in the same table. We find good agreement between individual $z_{\text {spec }}$ and $z_{\text {phot }}$ values, with a typical error $\left\langle\left|z_{\text {phot }}-z_{\text {spec }}\right|\right\rangle=0.15$ or $\left\langle\left|\frac{z_{\text {phot }}-z_{\text {spec }}}{1+z_{\text {spec }}}\right|\right\rangle=0.047$ and no catastrophic measurements. This strong consistency mainly stems from the use of space-based images of very similar PSF size, together with ground-based nearinfrared images obtained with MOIRCS in excellent seeing conditions $\left(0.4^{\prime \prime}\right)$.

\section{Results}

\subsection{Multiple images redshifts and identifications}

Figure 1 presents a color image of the central region of Abell 1703, combining the $B, V$, and $Z A C S$ filters, where we marked in white the location of the 11 multiply imaged sources identified in our previous work (L08). Our spectroscopic data enabled us to measure the redshifts for the following 8 systems (displayed as white squares in Fig. 1):

- system 1 at $z=0.8889$ forms a very highly magnified ringshaped configuration with 4 images close to the BCG and a less-magnified 5th image to the south west. Although its redshift was previously derived in L08, we obtained a new spectrum with the same mask in much better conditions;

- system 3 at $z=3.277$ is located to the north of the BCG, with 3 images: 2 bright merging images and a fainter counterimage to the west;

- systems $4 / 5$ at $z=1.9082$ are two individual regions identified in the same source, which forms a cusp configuration of 3 images; 

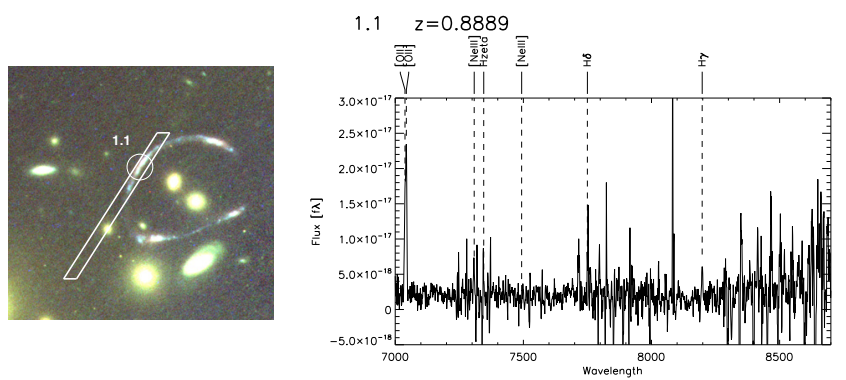

$3.3 \quad z=3.2770$
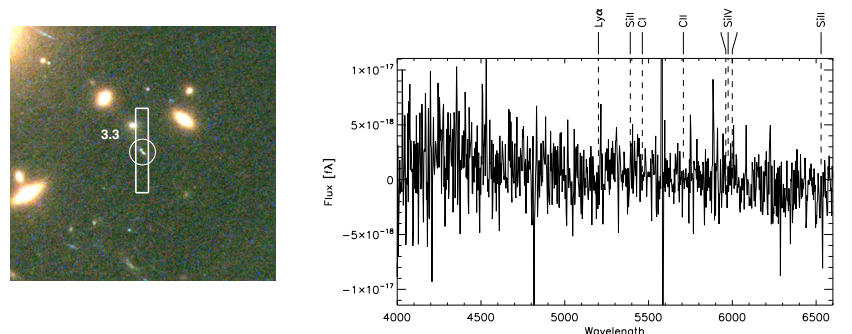

$6.1 \quad z=2.3600$
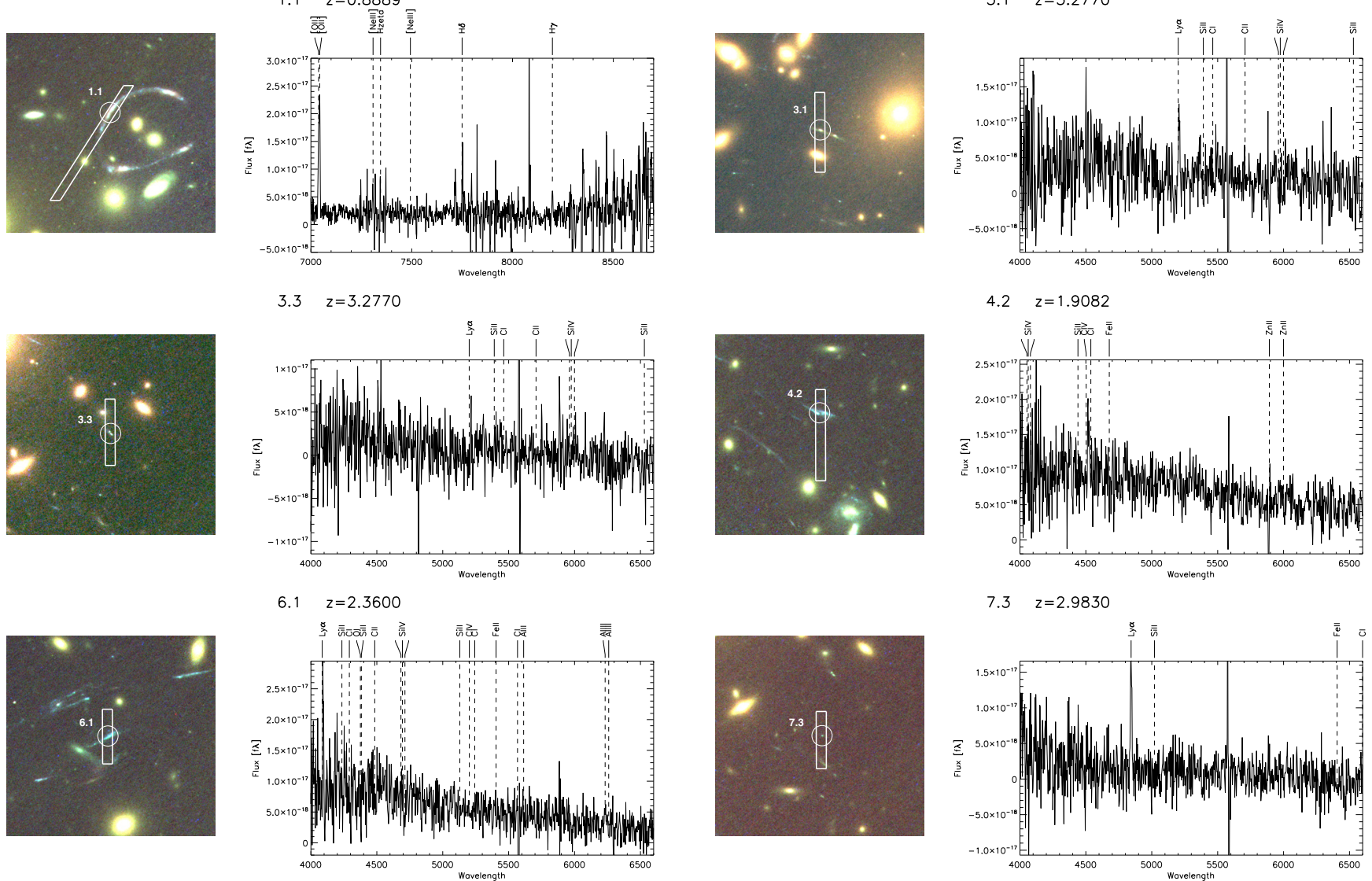

$7.3 \quad z=2.9830$

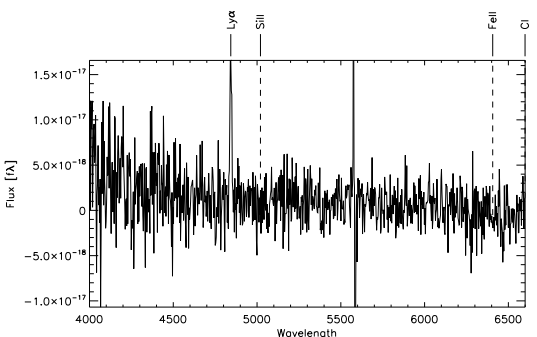

$11.3 \quad z=2.6270$
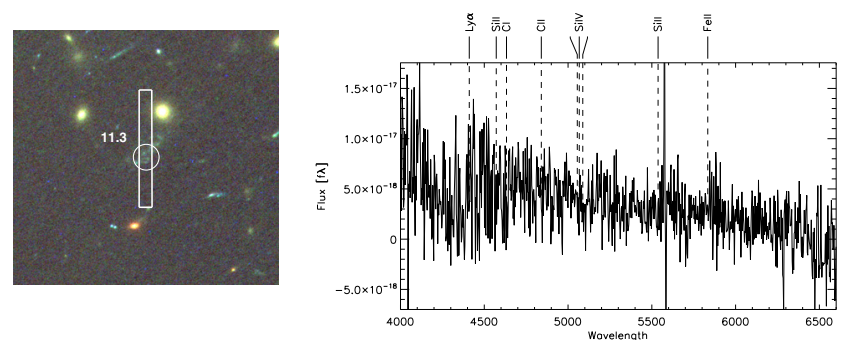

$15.3 \quad z=2.3550$
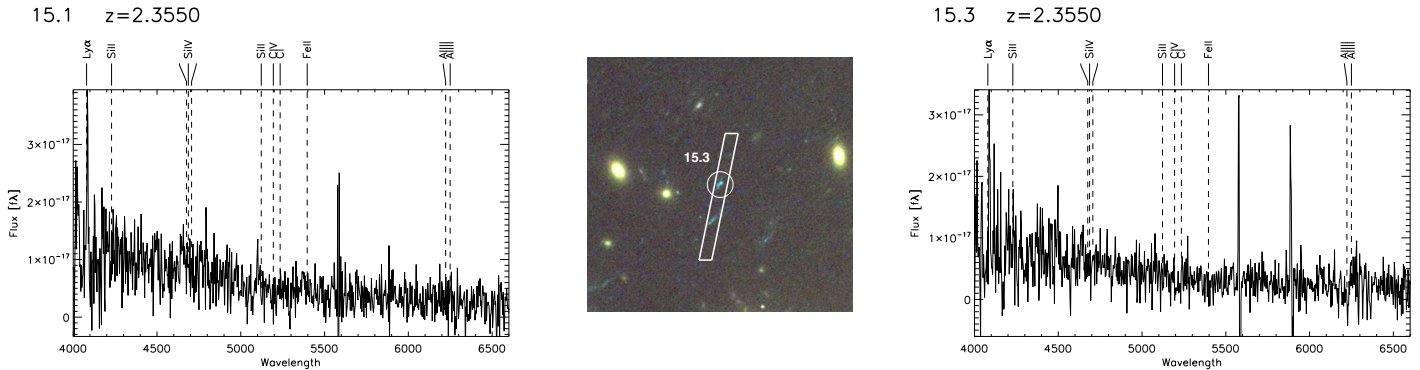

$20 z=1.2790$
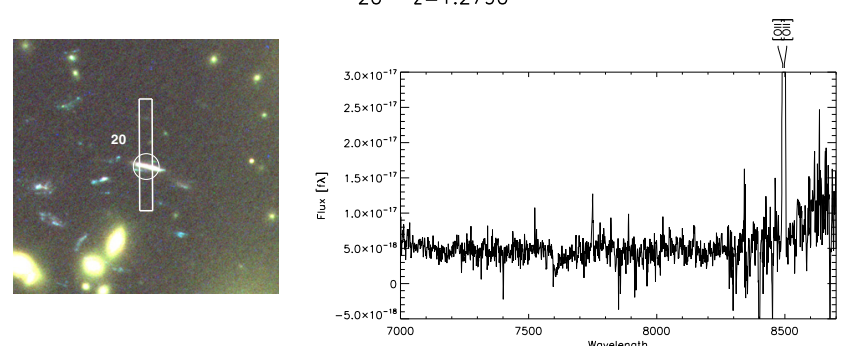

Fig. 2. BVIACS color image close-up on individual $z>0.5$ sources in the LRIS mask with the location of the slit, and corresponding extracted spectra. The $i$-band dropout (23, see Sect. 3.2) is presented separately in Fig. 4. 
$21 \quad z=1.2790$
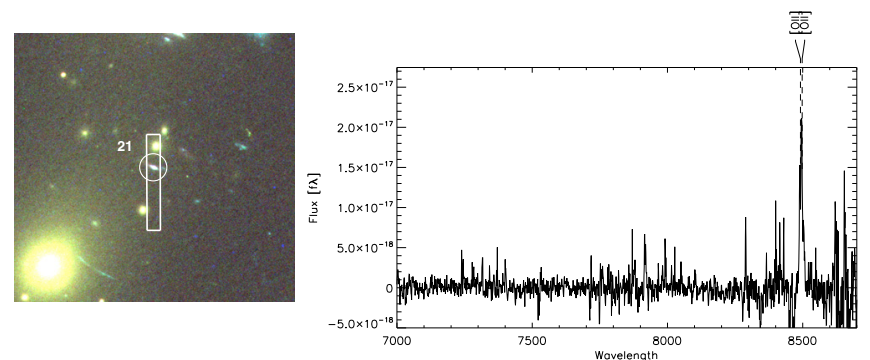

gal4 $z=3.2269$
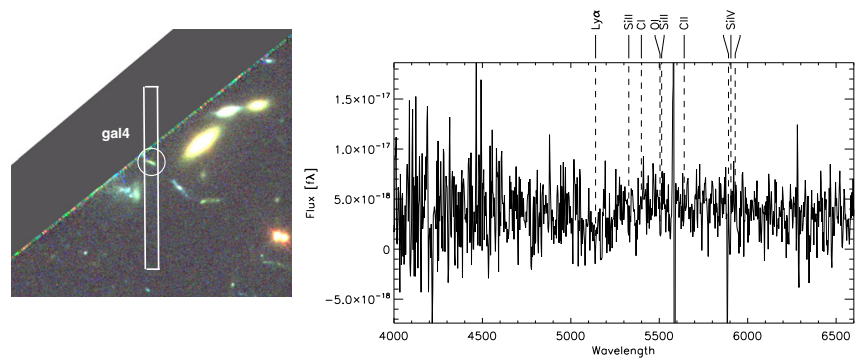

gal12 $z=2.2900$
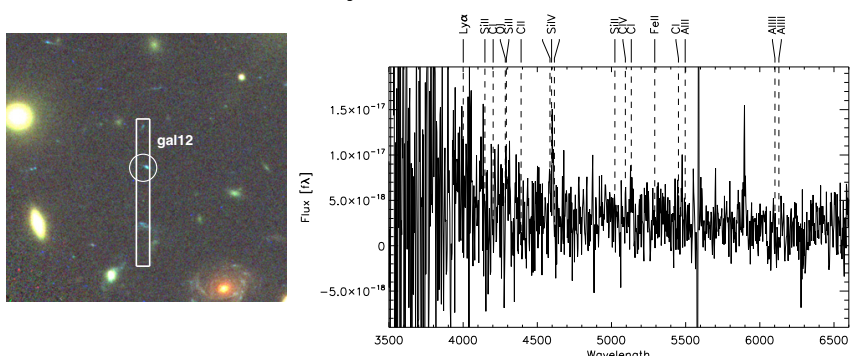

gal17 $z=1.2360$
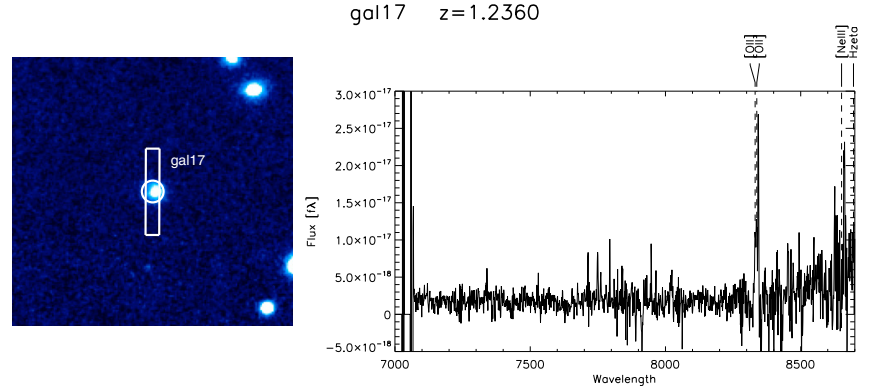

Fig. 3. Continuation of Fig. 2.

- system 6 at $z=2.360$ is a similar configuration as systems $4 / 5$, but closer to the center of the cluster;

- system 7 at $z=2.983$ is again a cusp configuration like systems $4 / 5$, but farther from the center of the cluster;

- systems $10 / 11$ at $z=2.627$ are two individual regions identified in the giant tangential arc to the south east of the BCG. A fainter counter-image is located to the west. The new mass model presented here enabled us to associate the radial feature reported in L08, very close to the BCG (next to the ring configuration of system 1 ) to be a 4th image (10.4) associated with this arc;

- system 15 at $z=2.355$ is a typical Einstein-cross configuration of 4 images surrounding the BCG. We note that systems $6(z=2.360)$ and $15(z=2.355)$ have similar redshifts and are therefore very close to one another in the source plane, with a projected separation of $67 \pm 6 \mathrm{kpc}$;
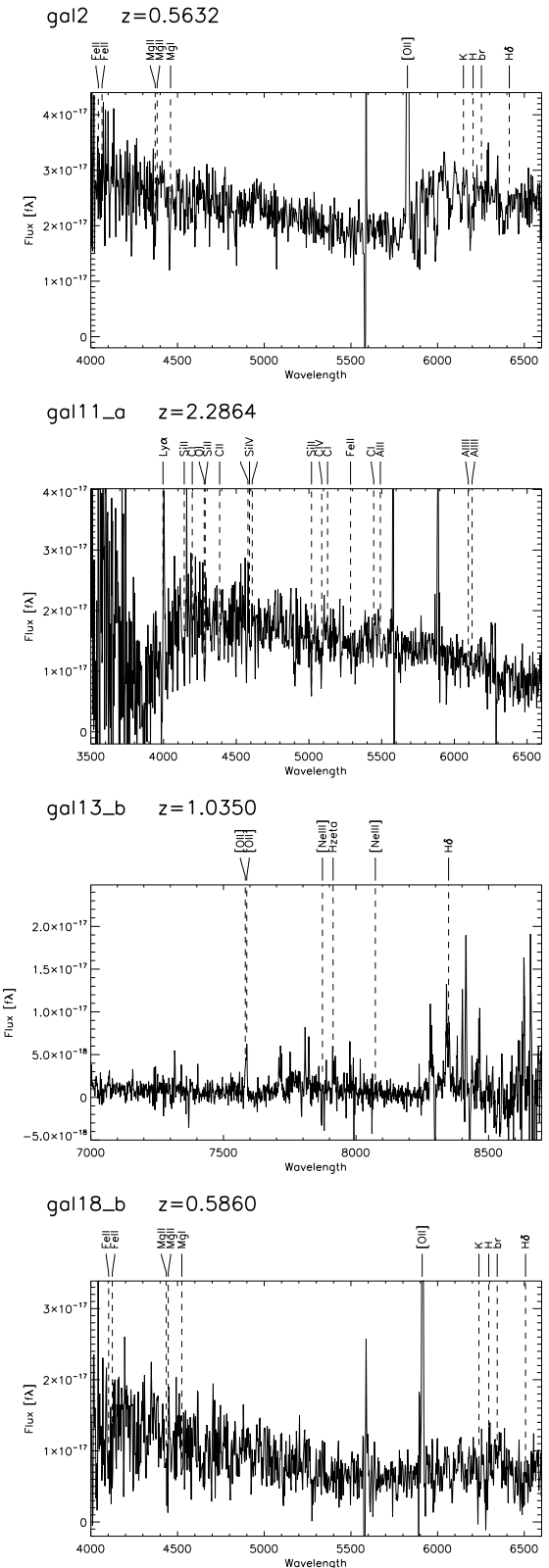

- system 16 at $z=2.810$ is another Einstein-cross configuration located at a slightly higher redshift, but found very close to system 15 in the image plane due to projection effects.

Additional sources 20 and 21, which were included in the mask as a possible new multiple system, show a very similar spectroscopic redshift $z=1.279$ with [OII] doublet of emission lines. However, they are located too far from the center of the cluster to be multiply imaged at this redshift.

Apart from system 1, all the other systems are located in the redshift range $1.9<z<3.3$. Using the new spectroscopic redshifts into the mass model (see Sect. 3.3), we have computed the predicted region of multiple images for a redshift $z=6$ (Fig. 1). As we expect all the multiple images present in the HST optical bands to lie within this region, we use it to update our systematic search for multiple images based on the photometric redshift catalog. 
Table 2. Overview of the sources included in the LRIS mask.

\begin{tabular}{|c|c|c|c|c|c|c|c|c|}
\hline ID & $\alpha(\mathrm{J} 2000.0)$ & $\delta(\mathrm{J} 2000.0)$ & $z_{\text {spec }}$ & $z_{\text {phot }}$ & $F 775 W$ & $\mu$ (mags) & $z_{\text {class }}$ & Features \\
\hline 1.1 & 198.77725 & 51.81934 & $0.8889 \pm 0.0003$ & $0.965_{-0.240}^{+0.075}$ & $22.19 \pm 0.01$ & $4.78 \pm 0.22$ & 4 & {$[\mathrm{OII}],[\mathrm{NeIII}], \mathrm{H} \delta, \mathrm{H} \gamma$} \\
\hline 3.1 & 198.76696 & 51.83205 & $3.277 \pm 0.002$ & $3.35_{-0.134}^{+0.052}$ & $22.69 \pm 0.02$ & $3.40 \pm 0.47$ & 4 & $L y_{\alpha}(\mathrm{em})$, SiIV, SiII \\
\hline 3.3 & 198.75824 & 51.82982 & $3.277 \pm 0.002$ & $3.35_{-0.036}^{+0.024}$ & $25.34 \pm 0.05$ & $1.66 \pm 0.22$ & 4 & $L y_{\alpha}(\mathrm{em})$, SiIV, SiII \\
\hline $4.2 / 5.2$ & 198.76075 & 51.82487 & $1.9082 \pm 0.004$ & $2.25_{-0.222}^{+0.036}$ & $23.72 \pm 0.02$ & $2.54 \pm 0.03$ & 3 & CIV, SiII, ZnII \\
\hline 6.1 & 198.77984 & 51.82640 & $2.360 \pm 0.002$ & $2.59_{-0.159}^{+0.0222}$ & $23.56 \pm 0.02$ & $1.96 \pm 0.04$ & 4 & $L y_{\alpha}(\mathrm{em})$, SiIV, CIV, SiII \\
\hline 7.3 & 198.75869 & 51.82814 & $2.983 \pm 0.002$ & $3.20_{-0.675}^{+0.1210}$ & $26.81 \pm 0.10$ & $1.87 \pm 0.02$ & 9 & $L y_{\alpha}(\mathrm{em})$ \\
\hline 10.1 & 198.78708 & 51.81424 & $2.627 \pm 0.004$ & $3.10_{-0.162}^{+0.032}$ & $21.40 \pm 0.01$ & $2.69 \pm 0.65$ & 3 & $L y_{\alpha}(\mathrm{abs}), \mathrm{SiIV}$ \\
\hline 11.3 & 198.76242 & 51.80954 & $2.627 \pm 0.004$ & $2.70_{-0.261}^{+0.189}$ & $23.55 \pm 0.02$ & $1.73 \pm 0.02$ & 3 & $L y_{\alpha}(\mathrm{abs}), \mathrm{SiIV}, \mathrm{SiII}$ \\
\hline 15.1 & 198.76284 & 51.81246 & $2.355 \pm 0.002$ & $2.44_{-0.231}^{+0.171}$ & $24.89 \pm 0.05$ & $2.26 \pm 0.10$ & 4 & $L y_{\alpha}(\mathrm{em}), \mathrm{CIV}$ \\
\hline 15.3 & 198.78825 & 51.82173 & $2.355 \pm 0.002$ & $2.60_{-0.363}^{+0.150}$ & $25.44 \pm 0.05$ & $1.56 \pm 0.05$ & 4 & $L y_{\alpha}(\mathrm{em}), \mathrm{SiIV}, \mathrm{CIV}$ \\
\hline $16.1 / 16.3$ & 198.78851 & 51.82096 & $2.810 \pm 0.004$ & $2.75_{-0.165}^{+0.173}$ & $25.52 \pm 0.06$ & $1.61 \pm 0.02$ & 3 & $L y_{\alpha}(\mathrm{abs}), \mathrm{FeII}$ \\
\hline 20 & 198.76659 & 51.83626 & $1.279 \pm 0.0005$ & $1.10_{-0.126}^{+0.125}$ & $21.86 \pm 0.01$ & $1.51 \pm 0.03$ & 4 & {$[\mathrm{OII}]$} \\
\hline 21 & 198.76000 & 51.83451 & $1.279 \pm 0.0005$ & $1.13_{-0.103}^{+0.130}$ & $22.57 \pm 0.02$ & $1.75 \pm 0.02$ & 4 & [OII $]$ \\
\hline 23 & 198.75612 & 51.80736 & $5.827 \pm 0.0015$ & $6.0_{-0.38}^{+0.42}$ & $27.20 \pm 0.30$ & $1.20 \pm 0.03$ & 9 & $L y_{\alpha}(\mathrm{em})$ \\
\hline gal2 & 198.78811 & 51.84276 & $0.5632 \pm 0.0004$ & $0.550_{-0.140}^{+0.123}$ & $20.49 \pm 0.01$ & $0.29 \pm 0.01$ & 4 & [OII], K, H, MgI, MgII \\
\hline gal4 & 198.76596 & 51.85902 & $3.2269 \pm 0.002$ & $3.44_{-0.07}^{+0.09}$ & $24.16 \pm 0.03$ & $0.40 \pm 0.02$ & 3 & $L y_{\alpha}(\mathrm{abs})$, SiII, SiIV \\
\hline gall1-a & 198.76772 & 51.79678 & $2.2864 \pm 0.002$ & $2.56_{-0.252}^{+0.235}$ & $22.86 \pm 0.01$ & $0.85 \pm 0.02$ & 4 & $L y_{\alpha}(\mathrm{em})$, SiII, CIV \\
\hline gal12 & 198.77770 & 51.79462 & $2.290 \pm 0.002$ & $2.32_{-0.698}^{+0.252}$ & $25.32 \pm 0.08$ & $0.83 \pm 0.03$ & 4 & $L y_{\alpha}(\mathrm{em}), \mathrm{CIV}, \mathrm{AlIII}$ \\
\hline gal13-b & 198.77518 & 51.78871 & $1.035 \pm 0.001$ & $1.05_{-0.112}^{+0.108}$ & $23.45 \pm 0.03$ & $0.48 \pm 0.01$ & 4 & {$[\mathrm{OII}]$} \\
\hline gal16 & 198.79701 & 51.77606 & $0.3906 \pm 0.0005$ & - & & $0.10 \pm 0.01$ & 4 & $\mathrm{~K}, \mathrm{H}$ \\
\hline gal17 & 198.79065 & 51.77097 & $1.236 \pm 0.0005$ & - & & $0.27 \pm 0.02$ & 4 & [OII $]$ \\
\hline gal18-b & 198.78816 & 51.77364 & $0.5860 \pm 0.0005$ & - & & $0.19 \pm 0.01$ & 4 & [OII], MgII, K, H \\
\hline gal19-a & 198.77100 & 51.76841 & $0.2909 \pm 0.0005$ & - & & $0.01 \pm 0.01$ & 4 & $\mathrm{~K}, \mathrm{H}$ \\
\hline gal19-b & 198.77063 & 51.76935 & $0.3622 \pm 0.0005$ & - & & $0.06 \pm 0.01$ & 4 & {$[\mathrm{OII}], \mathrm{K}, \mathrm{H}$} \\
\hline gal1 & 198.78155 & 51.83971 & $0.1432 \pm 0.0005$ & & & & 4 & {$[\mathrm{OII}], \mathrm{H} \beta,[\mathrm{OIII}]$} \\
\hline gal3 & 198.77735 & 51.85023 & $0.087 \pm 0.0005$ & & & & 4 & {$[\mathrm{OII}], \mathrm{K}, \mathrm{H}, \mathrm{H} \beta$, [OIII] } \\
\hline gal11-b & 198.76774 & 51.79744 & $0.280 \pm 0.001$ & & & & 4 & {$[\mathrm{OII}], \mathrm{H} \beta,[\mathrm{OIII}]$} \\
\hline gal13-a & 198.77540 & 51.78808 & $0.1762 \pm 0.0005$ & & & & 4 & $\mathrm{~K}, \mathrm{H}$ \\
\hline gal14 & 198.78653 & 51.78574 & $0.1011 \pm 0.0003$ & & & & 4 & {$[\mathrm{OII}], \mathrm{H} \beta,[\mathrm{OIII}]$} \\
\hline gal18-a & 198.78753 & 51.77299 & $0.2705 \pm 0.0005$ & & & & 4 & $\mathrm{~K}, \mathrm{H}$ \\
\hline gal20 & 198.79633 & 51.76602 & $0.2766 \pm 0.0005$ & & & & 4 & $\mathrm{~K}, \mathrm{H}$ \\
\hline gal21 & 198.78704 & 51.76265 & $0.2781 \pm 0.0005$ & & & & 4 & $\mathrm{~K}, \mathrm{H}$ \\
\hline gal22-a & 198.77246 & 51.75876 & $0.2410 \pm 0.0005$ & & & & 4 & {$[\mathrm{OII}], \mathrm{K}, \mathrm{H}$} \\
\hline gal22-b & 198.77236 & 51.76022 & $0.2766 \pm 0.0005$ & & & & 4 & $\mathrm{~K}, \mathrm{H}$ \\
\hline gal23 & 198.75441 & 51.86258 & $0.1765 \pm 0.0004$ & & & & 4 & {$[\mathrm{OII}], \mathrm{K}, \mathrm{H}, \mathrm{H} \beta,[\mathrm{OIII}]$} \\
\hline gal24 & 198.75110 & 51.86508 & $0.2683 \pm 0.0005$ & & & & 4 & $\mathrm{~K}, \mathrm{H}$ \\
\hline
\end{tabular}

From left to right: identification, astrometric position, spectroscopic redshift, photometric redshift estimate, ACS-F775W band photometry when available, magnification in magnitudes, redshift quality class (see Sect. 2.3), main spectroscopic features. The mentions (abs) and (em) refer to an $\mathrm{Ly}_{\alpha}$ line in absorption or in emission, respectively. The first part of the table corresponds to multiply-imaged systems, the second part to background sources, the third part to cluster members and foreground sources.

We find three additional systems complementing the list presented in L08. We display them in Fig. 1 and report the corresponding positions and photometric redshifts in Table 3:

- system 12 is again a typical cusp configuration of 3 images located to the north and "following" the nearby systems $4 / 5$, 7, 8 and 9;

- systems 13 and 14 are two other Einstein-cross configurations of 4 images surrounding the BCG. System 14 is located near systems 15 and 16 in the image plane, while system 13 is closer to the cluster center.

We also report in Table 3 the same parameters for the three remaining systems from L08 (systems 2, 8 and 9), used in the mass model but lacking spectroscopic redshifts. The redshift of 10.4 has a spectroscopic measurement and therefore is not a free parameter in the model. System 14 and image 9.1 are too faint to provide a reliable photometric redshift estimate.

\subsection{Spectroscopy of a remarkably bright i-dropout}

One of the sources in the LRIS mask, identified as 23 in the spectroscopic catalog (Table 2), deserves particular attention. Located to the south west of the cluster center (green triangle in Fig. 1), it has been selected for its very red $i-z=2.9 \pm 0.3$ color and bright $z=24.30 \pm 0.07$, while undetected blueward of the $i$ band in the other deep ACS images. The NICMOS and MOIRCS photometry $J=23.93 \pm 0.07$ and $H=23.75 \pm 0.10$ further constrain its spectral energy distribution to be compatible with a photometric redshift $z_{\text {phot }}=6.0 \pm 0.42$ (Fig. 4, top and middle panels), and the $i-z$ color make it a very strong $i$-dropout photometric candidate according to the Bouwens et al. (2006) selection criterion.

The LRIS spectrum for this object covers the wavelength range 3800-9230 $\AA$, corresponding to $2.12<z<6.59$ for Lyman- $\alpha$. We detect a $5 \sigma$-significant emission line at 
Table 3. Location and redshift estimate of three new multiply-image systems, as well as the radial feature (10.4) associated with the giant arc (systems 10 and 11).

\begin{tabular}{lccllll}
\hline \hline ID & $\alpha(\mathrm{J} 2000.0)$ & $\delta(\mathrm{J} 2000.0)$ & $z_{\text {model }}$ & $z_{\text {phot }}$ & $F 775 W$ & $\mu$ (mags) \\
\hline New multiple images & & & & & \\
10.4 & 198.77173 & 51.81906 & $z_{\text {spec }}=2.627$ & - & $24.77 \pm 0.08$ & $1.55 \pm 0.17$ \\
12.1 & 198.77456 & 51.829946 & $3.31 \pm 0.16$ & $3.33_{-0.32}^{+0.47}$ & $26.68 \pm 0.10$ & $1.93 \pm 0.07$ \\
12.2 & 198.76568 & 51.828658 & & $3.35_{-0.31}^{+0.22}$ & $24.47 \pm 0.04$ & $1.87 \pm 0.16$ \\
12.3 & 198.75867 & 51.826482 & & $3.16_{-0.52}^{+0.24}$ & $26.33 \pm 0.08$ & $2.17 \pm 0.04$ \\
13.1 & 198.78238 & 51.821204 & $0.839 \pm 0.007$ & $1.15_{-0.16}^{+0.13}$ & $26.93 \pm 0.11$ & $1.48 \pm 0.03$ \\
13.2 & 198.76796 & 51.818374 & & $0.68_{-0.12}^{+0.46}$ & $24.18 \pm 0.04$ & $2.42 \pm 0.33$ \\
13.3 & 198.77204 & 51.814973 & & $1.13_{-0.10}^{+0.08}$ & $26.29 \pm 0.08$ & $2.16 \pm 0.19$ \\
13.4 & 198.76692 & 51.815130 & & $1.07_{-0.10}^{+0.19}$ & $25.12 \pm 0.05$ & $2.69 \pm 0.06$ \\
14.1 & 198.78662 & 51.820542 & $1.58 \pm 0.02$ & - & $25.55 \pm 0.11$ & $1.63 \pm 0.02$ \\
14.2 & 198.76868 & 51.820191 & & - & $25.40 \pm 0.17$ & $1.26 \pm 0.48$ \\
14.3 & 198.76447 & 51.812192 & & - & $26.33 \pm 0.16$ & $2.13 \pm 0.03$ \\
14.4 & 198.77540 & 51.811945 & & - & $26.30 \pm 0.15$ & $0.81 \pm 0.10$ \\
\hline Other multiple systems from L08 & & & & \\
2.2 & 198.77156 & 51.81174 & $2.16_{? 0.09}^{+0.15}$ & $2.31_{-0.38}^{+0.46}$ & $25.29 \pm 0.08$ & $4.45 \pm 0.18$ \\
2.3 & 198.76970 & 51.81186 & & $2.23_{-0.66}^{+0.19}$ & $26.15 \pm 0.10$ & $4.37 \pm 0.16$ \\
8.1 & 198.77250 & 51.83045 & $2.933_{-0.077}^{+0.004}$ & $2.80_{-0.09}^{+0.17}$ & $24.95 \pm 0.06$ & $2.46 \pm 0.18$ \\
8.2 & 198.76608 & 51.82949 & & $2.77_{-0.11}^{+0.21}$ & $23.06 \pm 0.02$ & $2.19 \pm 0.10$ \\
8.3 & 198.75863 & 51.82740 & & $2.72_{-0.12}^{+0.20}$ & $25.07 \pm 0.06$ & $2.00 \pm 0.03$ \\
9.1 & 198.77176 & 51.83030 & $3.271_{-0.124}^{+0.031}$ & - & $26.68 \pm 0.15$ & $3.09 \pm 0.20$ \\
9.2 & 198.76690 & 51.82957 & & $2.995_{-0.378}^{+0.195}$ & $25.32 \pm 0.08$ & $2.48 \pm 0.19$ \\
9.3 & 198.75813 & 51.82708 & & $3.00_{-0.60}^{+0.37}$ & $26.92 \pm 0.12$ & $1.98 \pm 0.02$ \\
\hline
\end{tabular}

From left to right: identification, astrometric position, redshift estimate from the lensing model, photometric redshift estimate, magnification in magnitudes. We also report the same values for the three remaining systems from L08 (systems 2, 8 and 9), used in the mass model but lacking spectroscopic redshifts. The redshift of 10.4 has a spectroscopic measurement and therefore is not a free parameter in the model. System 14 and image 9.1 are too faint to provide a reliable photometric redshift estimate.

$\lambda=8300.5 \AA$ (Fig. 4, lower panel), with an integrated flux $f=2.5 \pm 0.4 \times 10^{-17} \mathrm{erg} \mathrm{s}^{-1} \mathrm{~cm}^{-2}$, and an observed equivalent width $W>50 \AA$ when assuming a $5 \sigma$ upper limit on the underlying continuum. This line is slightly resolved in the spectral direction, with a possibly asymmetric profile. To estimate this asymmetry, we used the weighted skewness statistics $S_{w}$, which is based on the third momentum of the flux distribution $f_{i}$ (seen as an array of size $\mathrm{N}$ ) and was used as a selection criterion for Lyman- $\alpha$ emitters (LAEs, Kashikawa et al. 2006). The value of $S_{w}$, measured in Angstroms, is defined as

$S_{w}=\left(\frac{1}{I \sigma^{3}} \sum_{1}^{N}\left(x_{i}-\bar{x}\right)^{3} f_{i}\right)\left(\lambda_{10, r}-\lambda_{10, b}\right)$

where $\left(x_{i}\right)$ and $\left(f_{i}\right)$ are the arrays of coordinates and fluxes, of size $N, I=\sum_{i}^{N} f_{i}$, and $(\bar{x}, \sigma)$ represent the mean and standard deviation of the array $\left(x_{i}\right)$, respectively. The weight factors $\lambda_{10, r}$ and $\lambda_{10, b}$ correspond to the wavelengths where the flux drops to $10 \%$ of its peak value on the red and blue sides of the line emission (see Kashikawa et al. 2006, for a more detailed discussion about the use of $S_{w}$ ). In our case, we measure $\lambda_{10, r}=8305 \AA$ and $\lambda_{10, b}=8298 \AA$ on the spectrum of the dropout, and a corresponding $S_{w}=5.6 \pm 1.4 \AA$. The error on $S_{w}$ has been measured using a bootstrap resampling of the fluxes. The skewness value is slightly superior to the critical value $S_{w}=3 \AA$ which has been used as a lower limit for selecting single line emitters as LAEs in Kashikawa et al. (2006) and Shimasaku et al. (2006). The single emission line is therefore likely to be Lyman- $\alpha$ at a redshift $z=5.827$, so compatible with the photometric redshift prediction.
At this redshift, we compute a magnification factor and associated error $\mu=1.2 \pm 0.03 \mathrm{mag}$, derived with the improved strong lensing model (see Sect. 3.3.2). We note that, although this object is a single image, it is located very close to the region of strong lensing, allowing an accurate estimate of the magnification with our model.

After correcting for this magnification, the source is both magnified and intrinsically bright $\left(H_{\text {unlensed }}=24.9\right)$, as there are only 9 of such sources at $z \sim 6$ brighter than $z=25.5 \mathrm{AB}$ found in the UDF, HUDF and GOODS fieds (Bouwens et al. 2006). This object is similar in redshift and intrinsic magnitude to the spectroscopically confirmed $i$-dropouts by Stanway et al. (2004) at $z=5.78$ and $z=5.83$, or the slightly magnified object at $z=5.515$ found in the cluster RDCS1252.9-292 (Dow-Hygelund et al. 2005). Based on the latest constraints of the luminosity function at $z \sim 6$ (Bouwens et al. 2008), the unlensed magnitude corresponds to an $3.6 L^{*}$ galaxy. We also note that the very red $i-z=2.9$ color is much redder than for any of the 9 dropouts with $z<25.5$ from Bouwens et al. (2006), detected in both the $i$ and $z$ bands, and where the reddest color was found to be $i-z=2.4$. Thanks to the magnification and the combination of filters at this redshift, the $i-z$ color allows the average depression factor $\langle D\rangle=\left\langle 1-f_{\text {obs }} / f_{\text {int }}\right\rangle$ at $z \sim 6$ to be measured, between the observed and intrinsic fluxes shortward of Lyman- $\alpha$, due to line blanketting. The corresponding value $\langle D\rangle \sim 0.96$ is a lower limit, due to possible contamination of the red side of the line in the $i$ band filter. This value is close (but slightly superior) to the predicted value at $z=6(\langle D\rangle=0.92)$ when following the Madau (1995) prescription.

Although the current photometry does not allow deriving physical parameters on this source (such as age, reddening, 

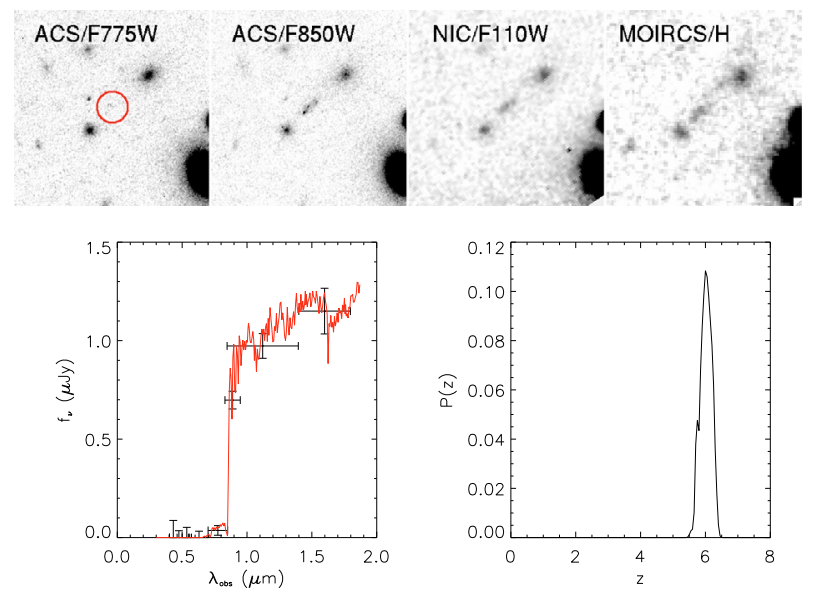

$23 \quad z=5.827$
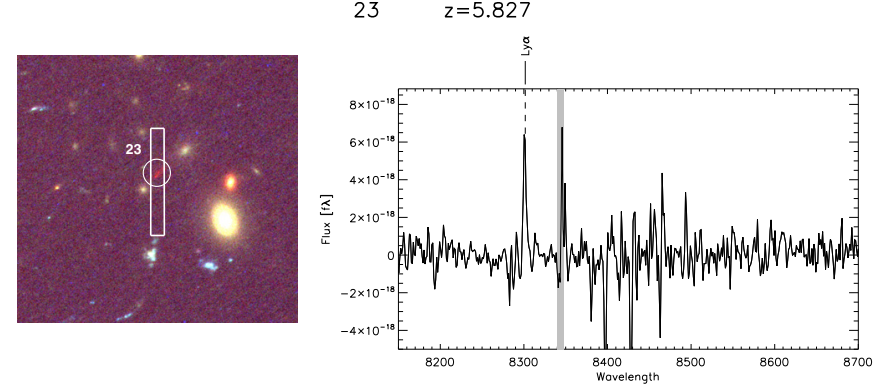

Fig. 4. A bright $i$-dropout found in the field of Abell 1703. The top panel shows the individual detections in the ACS, NICMOS, and MOIRCS images. The object is resolved and elongated along the shear direction, with an angular size $0.45^{\prime \prime}$. Middle panels: best fit of the spectral energy distribution with the photometric redshift software (left), and corresponding redshift probability distribution (right). Bottom panels: VIZACS color image with the location of the LRIS slit (left), and close-up on the extracted spectrum where we detected an emission line at $8301 \AA$ (right). The grey region highlights the location of a bright OH skyline.

stellar mass), when probing the rest-frame UV at $z=6$ we can measure the UV spectral slope $\beta$, defined as $f_{\lambda} \propto \lambda^{\beta}$. Using the $J-H=0.18$ color, we derive $\beta \sim-1.5$, a value redder than a spectrum flat in $f_{v}(\beta=-2.0)$, as can be seen in Fig. 4. Although this value is typical of Lyman-break galaxies at $z \sim 3$ $(\beta=-1.5 \pm 0.4$, Adelberger \& Steidel 2000), it is redder than for the sample of $27 i$-dropouts studied by Stanway et al. (2005), who derived $\beta \sim-2.0$ based on $J$ and $H$ photometry, or the sample of lensed $z$-band dropouts presented in Richard et al. (2008). The use of IRAC observations probing the longer wavelengths would allow us to understand whether this redder color stems from an old stellar population or an effect of reddening, similar to the work done by Egami et al. (2005) on a highly lensed galaxy at $z \sim 6.8$ discovered in Abell 2218 (Kneib et al. 2004).

\subsection{Strong lensing}

\subsubsection{Modeling method}

Our starting point in modeling the dark matter distribution in Abell 1703 is the mass model presented in details by L08, where we used the LENSTOOL ${ }^{1}$ public software (Jullo et al. 2007) to constrain a parametric mass model with the identified multiple systems. The model is optimized through the new Bayesian Markov chain Monte-Carlo (hereafter MCMC)

\footnotetext{
${ }^{1}$ http://www . oamp.fr/cosmology/lenstool/
}

sampler, described in detail in Jullo et al. (2007). This process uses the observational constraints (positions of the multiply imaged systems) to optimize the parameters describing the mass distribution by matching the location of each image of a given system in the source plane.

The contribution of the stellar mass from the central galaxy is parametrized by a Pseudo Isothermal Elliptical Mass Distribution (PIEMD) using the profile derived from the photometry. The PIEMD profile, which has been widely used for modeling cluster-scale (Smith et al. 2005) and galaxy-scale (Natarajan et al. 1998) halos, assumes each dark matter clump can be parametrized by a central position, ellipticity $e^{2}$, position angle $\theta$, central velocity dispersion $\sigma_{0}$, and two characteristic radii: a core and a cut radius. The total mass is proportional to $r_{\text {cut }} \sigma_{0}^{2}$.

Using this parametrization, the PIEMD mass density $\Sigma(x, y)$ takes the form

$\Sigma(x, y)=\frac{\sigma_{0}^{2}}{2 G} \frac{r_{\text {cut }}}{r_{\text {cut }}-r_{\text {core }}}\left[\frac{1}{\left(r_{\text {core }}^{2}+\rho^{2}\right)^{1 / 2}}-\frac{1}{\left(r_{\text {cut }}^{2}+\rho^{2}\right)^{1 / 2}}\right]$

where $x$ and $y$ coordinates are oriented along the position angle $\theta, \rho^{2}=\left[\left(x-x_{\mathrm{c}}\right) /(1+\epsilon)\right]^{2}+\left[\left(y-y_{\mathrm{c}}\right) /(1-\epsilon)\right]^{2}, \epsilon=e /(2-e)$, and $\left(x_{\mathrm{c}}, y_{\mathrm{c}}\right)$ is the center of the mass distribution (see also Smith et al. 2005 for more details).

The cluster-scale dark-matter component is modeled with a generalized NFW (hereafter gNFW) profile (Zhao 1996) including a central logarithmic slope $\alpha$ :

$\rho(r)=\frac{\rho_{\mathrm{c}} \delta_{\mathrm{c}}}{\left(r / r_{\mathrm{s}}\right)^{\alpha}\left(1+\left(r / r_{\mathrm{s}}\right)\right)^{(3-\alpha)}}$

where $r_{\mathrm{s}}$ is a scale radius, $\rho_{\mathrm{c}}$ the critical density and $\delta_{\mathrm{c}}$ is related to the value of the concentration parameter $c_{200}$ through the relation:

$$
\delta_{\mathrm{c}}=\frac{200}{3} \frac{c_{200}^{3}}{\ln \left(1+c_{200}\right)-c_{200} /\left(1+c_{200}\right)} .
$$

Again, the spherical gNFW profile is generalized to an elliptical mass distribution using a relation similar to Eq. (2). A more detailed description of the implementation of the $\mathrm{gNFW}$ profile in lenstool is provided by Sand et al. (2008).

Individual cluster galaxies were added as small-scale PIEMD perturbers based on their photometry and shape parameters, using empirical scaling relations between their dynamical parameters (central velocity dispersion and scale radius) and their luminosity, assuming the Faber \& Jackson (1976) relation and a constant mass-to-light ratio for each galaxy (see Covone et al. 2006 for more details). The same parameters of one particular cluster galaxy to the north (852) were optimized individually to reproduce the configuration of the surrounding systems 3 , 7, 8, 9 and 12 .

We replaced the photometric redshifts kept as free parameters in L08 by their spectroscopic equivalents for the 7 relevant sources ( 9 systems). The precision of the new model, which uses the same parametrization as L08, can be estimated using the rms of the location of multiple images in the image plane, defined as

$$
\sigma_{i}=\sqrt{\sum_{j, k}\left(x \operatorname{obs}_{j, k}-x \operatorname{pred}_{j, k}\right)^{2}+\left(y \operatorname{obs}_{j, k}-y \operatorname{pred}_{j, k}\right)^{2}}
$$

\footnotetext{
${ }^{2}$ We use here the definition $e=1-b / a$ of the ellipticity, where $a$ and $b$ are the semi-major and semi-minor axis of the ellipse, respectively.
} 
Table 4. Best-fit parameters used to model the mass distribution.

\begin{tabular}{|c|c|c|c|c|c|c|c|c|c|}
\hline Clump & $(x)$ & (y) & $e$ & $\theta$ & $r(\mathrm{kpc})$ & $\alpha$ & $c_{200}$ & $\sigma_{0}\left(\mathrm{~km} \mathrm{~s}^{-1}\right)$ & $z_{\text {model }}$ \\
\hline NFW & $-0.79 \pm 0.08$ & $0.91 \pm 0.08$ & $0.230 \pm 0.006$ & $64.0 \pm 0.3$ & $476.9_{-42.8}^{+51.6}$ & $0.92_{-0.04}^{+0.05}$ & $4.72_{-0.45}^{+0.43}$ & - & - \\
\hline $\mathrm{cD}$ & {$[0.0]$} & {$[0.0]$} & [0.19] & {$[52]$} & {$[25]^{-42.8}$} & & & $355.7_{-12.8}^{+10.3}$ & - \\
\hline Galaxy 852 & [19.0] & {$[54.0]$} & {$[0.11]$} & {$[65.5]$} & $98.7 \pm 1.8$ & & & $320.9 \pm 3.5$ & - \\
\hline$L_{*}$ elliptical galaxy & & & & & $68.8_{-1.8}^{+0.2}$ & & & $202_{-3}^{+2}$ & - \\
\hline
\end{tabular}

Values in brackets were not optimized by the model (see text for details).

where $\left(x \mathrm{obs}_{j, k}, y \mathrm{obs}_{j, k}\right)$ and $\left(x_{\mathrm{pred}} \mathrm{j}_{j, k}, y\right.$ pred $\left._{j, k}\right)$ are the observed and predicted locations of image $j$ in system $k$, respectively. Using the exact same parametrization, we find a value of $\sigma_{i}=$ $1.3^{\prime \prime}$ similar to the result obtained in L08 $\left(1.4^{\prime \prime}\right)$. This precision is typical of strong lensing works using a similar number of multiples images (Richard et al. 2007; Elíasdóttir et al. 2007), and smaller by a factor of $\sim 2$ from the one obtained for Abell 1689 (2.87", Limousin et al. 2007).

We summarize the best-fit parameters of the new model in Table 4. In this table, the positions $(x)$ and $(y)$ of each clump are in arcsecs relative to the central galaxy $(\mathrm{RA}=13: 15: 05.276$, Dec $=+51: 49: 02.85$ ) and oriented in WCS (north is up, east is left). Orientations $\theta$ are measured in degrees and ellipticities $(e)$ of the potentials are given as $1-b / a$, where $a$ and $b$ are the semi-major and semi-minor axis of the ellipse, respectively. The radius $(r)$ refers to the scale radius $r_{\mathrm{s}}$ in the case of the gNFW profile, and the $r_{\text {cut }}$ radius in the case of the PIEMD profile (see L08 for details).

The main gNFW component shows a concentration $c_{200}=$ $4.72_{-0.45}^{+0.43}$, a scale radius $r_{\mathrm{s}}=476.9_{-42.8}^{+51.6} \mathrm{kpc}$, and an inner slope $\alpha=0.92_{-0.04}^{+0.05}$. The geometrical parameters (center, orientation and ellipticity) are very similar to the ones measured on the $\mathrm{cD}$ galaxy (see also Sect. 3.3.4 below). In comparison with the results from L08, the strongest variations in the best-fit parameters are found in the gNFW profile, which appears to be more concentrated (larger $c_{200}$ and smaller scale radius $r_{\mathrm{s}}$ ), but both results are compatible at the $3 \sigma$ level. Adding the new constraints (spectroscopic redshifts, new multiple systems), while keeping the same model parametrization, did not reduce the uncertainty in the best-fit parameters, even if both models reproduce the constraints on the multiple images with a similar precision. This argues for a remaining degeneracy within the gNFW parameters, which cannot be completely disentangled by a mass model purely based on strong lensing.

\subsubsection{Redshift predictions and magnifications}

One of the main benefits of the Bayesian approach is that the MCMC optimization provides a large number of models that sample the posterior probability-density function of all the parameters (Jullo et al. 2007). We can use these different realizations to estimate the average value and associated error for any given parameter or combination of parameters. In our case, we estimate the redshift and associated error for each of the new multiple systems 12,13 , and 14 . These values are reported in Table 3 . We also computed the magnification factors $\mu$ and associated error for every source in our LRIS mask (Table 2), as well as the other multiple systems (Table 3 ). The magnification factors range from $0.01-0.05$ mag for single images to about $4.8 \mathrm{mag}$ for the source 1.1 .

\subsubsection{Weak-lensing predictions}

Although solely based on strong lensing constraints out to $50^{\prime \prime}$ (the region of multiple images), our mass model can be used to

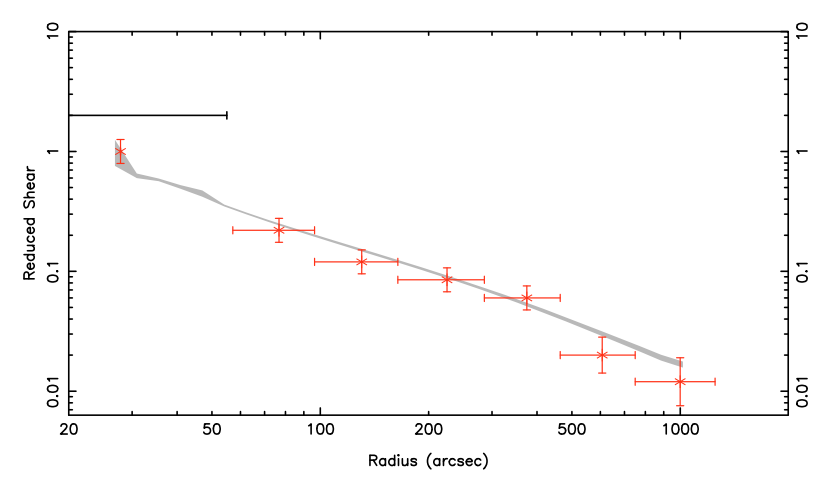

Fig. 5. Tangential reduced shear $g_{+}$predicted with the strong lensing model based on constraints in the central region (black error bar on top). The mean value and $3 \sigma$ error is presented as a function of radius in the grey-filled region. We overplot the datapoints obtained by Broadhurst et al. (2008) in their weak-lensing measurements with Subaru. Extrapolation of our strong lensing model forms a very good fit to these observations upto $r=1000 \operatorname{arcsec}$, or $4 \mathrm{Mpc}^{-1}$.

predict the tangential shear induced on background galaxies up to larger scales. The main observable with weak-lensing is the reduced shear $g=\gamma /(1-\kappa)$ where $\gamma$ is the gravitational shear and $\kappa$ the convergence. In order to compare our predictions with weak-lensing observations of Abell 1703 with Subaru described by Broadhurst et al. (2008), we adopt the definion of the tangential reduced shear $g_{+}$from Umetsu \& Broadhurst (2008), i.e. the projection of the shear perpendicularly to the direction of the cluster center (see also Medezinski et al. 2007).

Using the MCMC realizations, we determine predictions for $g^{+}$ and its associated 3- $\sigma$ error as a function of angular distance $r$ from the center of the mass distribution, assuming a distribution of background sources at a fixed redshift $z_{\mathrm{s}}=1.2$ corresponding the the mean of the redshift distribution of sources in the observations from Broadhurst et al. (2008). Figure 5 presents these estimations against their weak-lensing measurements over the same range of $r$. We find that our strong-lensing model represents a very good fit to the weak-lensing datapoints up to $4 \mathrm{Mpc} h^{-1}$, with a $\chi^{2}$ of $4.1 / 7=0.58$, even after extrapolating our mass model to a radial distance $20 \times$ larger than the region of strong lensing constraints. This argues again for the overall simplicity of the cluster, with a single NFW profile providing a good fit both for the strong-lensing and weak-lensing measurements.

\subsubsection{Ellipticity of the mass distribution and Einstein radius}

From the best-fit geometrical parameters found during the optimization of the mass model (Table 4), we notice a good agreement between the values for the ellipticity and position angle of the large scale gNFW profile $\left(e_{\mathrm{NFW}}=0.23, \theta_{\mathrm{NFW}}=63.9 \mathrm{de}-\right.$ grees) and the same parameters of the $\mathrm{cD}$ galaxy $\left(e_{\mathrm{cD}}=0.19\right.$, 
$\theta_{\mathrm{CD}}=52$ degrees) as measured with SExtractor ${ }^{3}$. This is not really surprising, as this correlation has previously been reported both in strong-lensing analysis (e.g. Gavazzi et al. 2003 in MS2137) and in X-ray observations (Hashimoto et al. 2008), as well as in numerical works (Dubinski 1998; Faltenbacher et al. 2005).

More generally, the total mass distribution of dark matter is the sum of this gNFW component and the various PIEMD profiles associated with the cluster galaxies, and the global ellipticity $e_{\text {mass }}$ may have a different value. We use the convergence map $\kappa$ (which shares an identical geometry with the mass distribution) to measure this ellipticity $e_{\text {mass }}$ a posteriori by fitting elliptical contours with the IRAF routine ellipse. We keep the center of the ellipses fixed at the peak of the mass map and let the ellipticity $e_{\text {mass }}(a)$ and position angle $\theta(a)$ vary as free parameters with the semi major axis $a$. In the central region $\left(a<15^{\prime \prime}\right)$, we find a best-fit value $e_{\text {mass }} \sim 0.23$ (Fig. 6) dominated by the geometry of the $\mathrm{cD}$ and the gNFW profile. At larger distances $e_{\text {mass }}(a)$ increases to reach a maximum of 0.4 at $a \sim 40^{\prime \prime}$ (Fig. 6), is due to an alignment of bright galaxies (as seen in projection) to the north west and the south east, therefore along a similar direction as $\theta_{\mathrm{NFW}}$. These galaxies contribute to increasing the value of $e_{\text {mass }}$ compared to $e_{\mathrm{NFW}}$, this effect being significant on the mass distribution up to the edge of the ACS field of view. The same ellipticity is seen on the weak-lensing reconstructions performed by Broadhurst et al. (2008).

We also computed the effective Einstein radius $R_{\mathrm{E}}$, defined as the radius $r$ from the center of the cluster (located at the peak of the mass distribution) at which $\bar{\kappa}\left(r<R_{\mathrm{E}}\right)=1$ (Broadhurst $\&$ Barkana 2008). We measured the value and error on $R_{\mathrm{E}}$ for the redshift $z=2.627$ of the giant tangential arc (formed with the systems 10 and 11), using the different MCMC realizations. We find $R_{\mathrm{E}}=28.0 \pm 0.25$ arcsec, a lower value than one would measure based on the simple distance of the giant arc (Fig. 6). This value is close but less than the estimate of $32^{\prime \prime}$ given by Broadhurst \& Barkana (2008), who mentions an agreement of $R_{\mathrm{E}}$ with the distance of the arc. This apparent discrepancy comes from the ellipticity of the mass distribution, as the giant arc is oriented along the major axis of the elliptical mass distribution. This ellipticity is now well-constrained by the large number of spectroscopically confirmed multiple images presented here, making the new measurement of $R_{\mathrm{E}}$ more robust.

We can use the same cumulative probability distribution $P\left(>R_{\mathrm{E}}\right)$ as Broadhurst \& Barkana (2008) to test the agreement of this new Einstein radius with the $\Lambda$ CDM Millennium simulation. This probability is computed from the distribution of concentrations at a given virial mass, accounting for the various biases arising from selecting a population of lensing clusters, as well as the projection effects. We find a higher probability $P=20 \%$ (instead of $7.9 \%$ ) of agreement, making Abell 1703 less discrepant than the other mentioned clusters (Abell 1689, CL0024 and RXJ1347) for its virial mass. Together with the results from these 3 other clusters $(P=8.5 \%, P=3.9 \%$, and $P=13 \%$, respectively) the combined probability for these 4 clusters is $9 e^{-5}$, or a $3.7 \sigma$ discrepancy. However, as mentioned earlier, there are few such clusters observed in the whole sky in comparison with the size of the Millennium Simulation, making it more plausible to reconcile the observations with whole-sky simulations.

\footnotetext{
3 The value of $e_{\mathrm{NFW}}$ given in L08 had an error in its definition and we update it to the correct one.
}

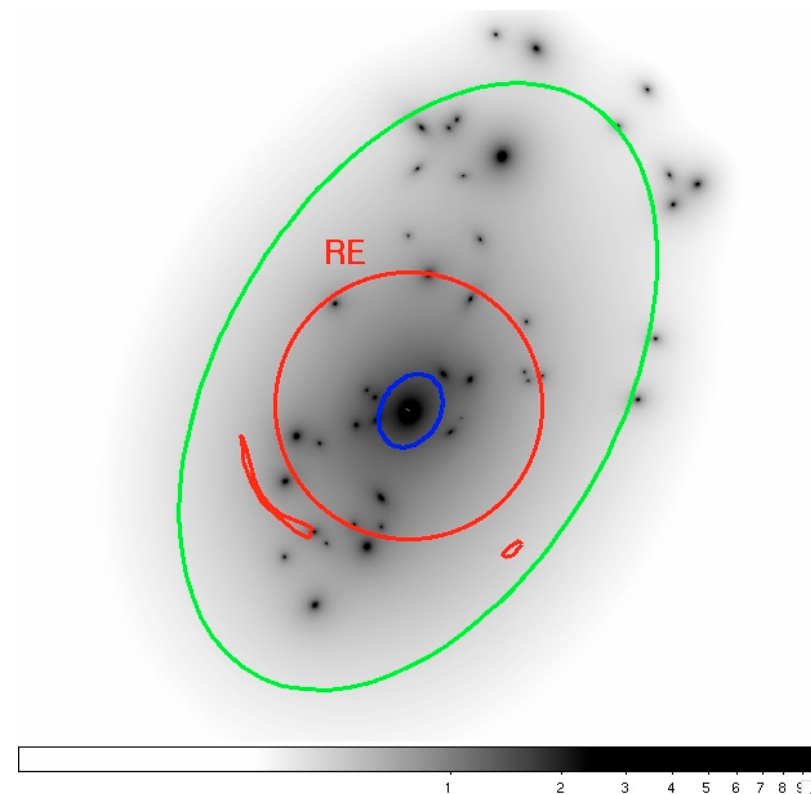

Fig. 6. Convergence map distribution $\kappa$ for a source at $z=2.627$, the redshift of the giant tangential arc (systems 10 and 11, located in red). By averaging this map inside increasing apertures, we measure the corresponding effective Einstein radius $R_{\mathrm{E}}$, slightly shorter than the distance to the giant arc due to the overall ellipticity of the mass distribution. The blue and green ellipses show the best-fit ellipses found on the overall mass distribution, with an increasing ellipticity when moving from small to large radii (see Sect. 3.3.4 for details).

\section{Abell 1703: a relaxed, unimodal cluster?}

Our strong-lensing analysis using a simple NFW component for the large-scale dark matter distribution, after individual treatment of the central cD galaxy and small-scale mass distributions associated with the galaxy substructure in the cluster, is able to reproduce the large number of multiple images identified in Abell 1703. Even if the overall rms in the predicted location of the multiple images $\left(1.3^{\prime \prime}\right)$ is still quite larger than the precision in astrometric measurements with HST (typically $0.1^{\prime \prime}$ ), we find no systematics as a function of the location of the multiples, which would argue for the need for secondary dark matter clumps. This was the case in the clusters Abell 2218 (Elíasdóttir et al. 2007) and Abell 68 (Richard et al. 2007), which show strong evidence of bimodality.

Furthermore, adding new spectroscopic measurements of 7 multiple sources has removed an identical number of free parameters (i.e., the redshifts of the sources) from the analysis presented in L08. However, we find no significant increase in the overall rms of the multiple images compared to this previous work, under the same assumptions. In addition, the strongest variations in the best-fit parameters compared with the previous values presented in L08 are found for the $\mathrm{gNFW}$ profile, but both results are compatible within the $3 \sigma$ confidence level. The remaining scatter in the positions of multiple images is very likely due to the small-scale substructure in the dark matter distribution, which is not included here apart from the visible cluster galaxies. One way to increase the complexity of this model would be to estimate small-scale deviations from the smooth mass distribution, using adaptive-grid based models (Jullo et al. 2009, submitted).

Finally, the best-fit parameters of our strong-lensing analysis have been consistent with weak-lensing measurements. Our best-fit concentration parameter $c_{200}=4.72_{-0.45}^{+0.43}$, 
corresponding to $c_{\mathrm{vir}}=5.82_{-0.56}^{+0.53}$ with the second definition based on the virial radius $r_{\mathrm{vir}}$, is somewhat lower than the value derived by Broadhurst et al. (2008), $c_{\mathrm{vir}}=9.92_{-1.63}^{+2.39}$, but marginally compatible at $2.4 \sigma$ level. Despite this lower concentration, the predicted shear profile provides an overall good fit to their observations, where the main difference is our smaller Einstein radius (which sets the location of the first datapoint in Fig. 5, as in Broadhurst et al. 2008), allowing for a shallower slope of the shear profile. We also note that the two shear measurements at large radii (where the signal gets weaker) are slightly lower than the predictions (but consistent within their error bars). Together, these differences affect the best-fit concentration parameter. The new Einstein radius measurement should be more reliable, since it is based on a larger number of multiple images at the center. There seems to be less discrepancy between our new strong-lensing NFW parameters and the weak-lensing measurements, as was the case for Abell 1689 (Broadhurst et al. 2005; Limousin et al. 2007).

Overall, we argue that these results provide further evidence that Abell 1703 is a relaxed cluster, as already suggested in L08. Follow-up X-ray observations of the cluster core should allow again this assumption to be confirmed, and would separate the $\mathrm{X}$-ray gas component from the dark matter in the mass distribution when doing the strong-lensing analysis, following the work by Bradač et al. (2008) for RXJ1347. Another independent measurement of the mass profile would be to study the dynamics of cluster galaxies up to large radii, giving another estimate of the virial radius and virial mass. Finally, we expect to find a larger sample of similar relaxed clusters from the ongoing LoCuSS survey (Zhang et al. 2008) of clusters with lower masses (as inferred from their X-ray luminosity) at $z \sim 0.2$. By pursuing the same strong lensing analysis on this broader sample, we will probe a wider distribution of Einstein radii, ellipticities, and concentration parameters with less bias compared to available numerical simulations.

Acknowledgements. We thank the anonymous referee for his/her helpful comments, and acknowledge useful discussions with Masamune Oguri, Carlos Frenk, and Mark Swinbank. J.R. acknowledges support from an European Union Marie-Curie Fellowship. M.L. acknowledges the Agence Nationale de la Recherche for its support, project number 06-BLAN-0067. The Dark Cosmology Center is funded by the Danish National Research Foundation. We thank the Danish Centre for Scientific Computing at the University of Copenhagen for providing a generous amount of time on its supercomputing facility. J.P.K. aknowledges the Centre National de la Recherche Scientifique for its support. The authors recognize and acknowledge the very significant cultural role and reverence that the summit of Mauna Kea has always had within the indigenous Hawaiian community. We are most fortunate to have the opportunity to conduct observations from this mountain.

\section{References}

Adelberger, K. L., \& Steidel, C. C. 2000, ApJ, 544, 218

Bolzonella, M., Miralles, J.-M., \& Pelló, R. 2000, A\&A, 363, 476

Bouwens, R. J., Illingworth, G. D., Blakeslee, J. P., \& Franx, M. 2006, ApJ, 653, 53

Bouwens, R. J., Illingworth, G. D., Franx, M., \& Ford, H. 2008, ApJ, 686, 230

Bradač, M., Schrabback, T., Erben, T., et al. 2008, ApJ, 681, 187

Broadhurst, T. J., \& Barkana, R. 2008, MNRAS, 390, 1647

Broadhurst, T., Benítez, N., Coe, D., et al. 2005, ApJ, 621, 53

Broadhurst, T., Umetsu, K., Medezinski, E., Oguri, M., \& Rephaeli, Y. 2008, ApJ, 685, L9

Covone, G., Kneib, J.-P., Soucail, G., et al. 2006, A\&A, 456, 409

Dow-Hygelund, C. C., Holden, B. P., Bouwens, R. J., et al. 2005, ApJ, 630, L137

Dubinski, J. 1998, ApJ, 502, 141

Egami, E., Kneib, J.-P., Rieke, G. H., et al. 2005, ApJ, 618, L5

Elíasdóttir, Á., Limousin, M., Richard, J., et al. 2007

[arXiv:astro-ph/0710.5636]

Faber, S. M., \& Jackson, R. E. 1976, ApJ, 204, 668

Faltenbacher, A., Allgood, B., Gottlöber, S., Yepes, G., \& Hoffman, Y. 2005, MNRAS, 362, 1099

Gavazzi, R., Fort, B., Mellier, Y., Pelló, R., \& Dantel-Fort, M. 2003, A\&A, 403, 11

Golse, G., Kneib, J.-P., \& Soucail, G. 2002, A\&A, 387, 788

Hashimoto, Y., Henry, J. P., \& Boehringer, H. 2008, MNRAS, 390, 1562

Hennawi, J. F., Gladders, M. D., Oguri, M., et al. 2008, AJ, 135, 664

Jullo, E., Kneib, J.-P., Limousin, M., et al. 2007, New J. Phys., 9, 447

Kashikawa, N., Shimasaku, K., Malkan, M. A., et al. 2006, ApJ, 648, 7

Kelson, D. D. 2003, PASP, 115, 688

Kneib, J.-P., Ellis, R. S., Smail, I., Couch, W. J., \& Sharples, R. M. 1996, ApJ, 471,643

Kneib, J.-P., Ellis, R. S., Santos, M. R., \& Richard, J. 2004, ApJ, 607, 697

Le Fevre, O., Crampton, D., Lilly, S. J., Hammer, F., \& Tresse, L. 1995, ApJ, 455,60

Limousin, M., Richard, J., Jullo, E., et al. 2007, ApJ, 668, 643

Limousin, M., Richard, J., Kneib, J.-P., et al. 2008, A\&A, 489, 23

Link, R., \& Pierce, M. J. 1998, ApJ, 502, 63

Madau, P. 1995, ApJ, 441, 18

Medezinski, E., Broadhurst, T., Umetsu, K., et al. 2007, ApJ, 663, 717

Natarajan, P., Kneib, J.-P., Smail, I., \& Ellis, R. S. 1998, ApJ, 499, 600

Navarro, J. F., Frenk, C. S., \& White, S. D. M. 1997, ApJ, 490, 493

Oguri, M., \& Blandford, R. D. 2009, MNRAS, 392, 930

Oke, J. B., Cohen, J. G., Carr, M., et al. 1995, PASP, 107, 375

Richard, J., Kneib, J.-P., Jullo, E., et al. 2007, ApJ, 662, 781

Richard, J., Stark, D. P., Ellis, R. S., et al. 2008, ApJ, 685, 705

Sand, D. J., Treu, T., Ellis, R. S., Smith, G. P., \& Kneib, J.-P. 2008, ApJ, 674, 711

Shimasaku, K., Kashikawa, N., Doi, M., et al. 2006, PASJ, 58, 313

Smith, G. P., Kneib, J.-P., Smail, I., et al. 2005, MNRAS, 359, 417

Stanway, E. R., Bunker, A. J., McMahon, R. G., et al. 2004, ApJ, 607, 704

Stanway, E. R., McMahon, R. G., \& Bunker, A. J. 2005, MNRAS, 359, 1184

Umetsu, K., \& Broadhurst, T. 2008, ApJ, 684, 177

Verdugo, T., de Diego, J. A., \& Limousin, M. 2007, ApJ, 664, 702

Zhang, Y.-Y., Finoguenov, A., Böhringer, H., et al. 2008, A\&A, 482, 451

Zhao, H. 1996, MNRAS, 278, 488 